\title{
Hermitian formulation of multiple scattering induced topological phases in metamaterial crystals
}

\author{
N. Kaina and R. Fleury $\odot$ \\ Laboratory of Wave Engineering, École Polytechnique Fédérale de Lausanne (EPFL), 1015 Lausanne, Switzerland
}

(Received 17 July 2020; accepted 17 September 2020; published 13 October 2020)

\begin{abstract}
In this article we develop an analog to the SSH model in tight-binding chains of resonators and an innovative Hermitian matrix formulation to describe the topological phases induced by multiple scattering at subwavelength scales in one-dimensional structured and locally resonant metamaterial crystals. We first start from a set of coupled dipole equations capturing the nature of the wave-matter interactions, i.e., hybridization between locally dispersive resonances and a continuum as well as infinite long range multiple scattering coupling, to analytically derive a matrix operator $H_{\mathrm{MS}}$, which is found to be Hermitian when evaluated on propagative bands. This new operator straightforwardly highlights how the composition, structure, scattering resonance, and Bloch periodicity together set in the chain macroscopic properties, in particular its topology. We analytically confirm the existence of a structure based topological transition in chiral-symmetric biperiodic metamaterial crystal chains, characterized by a winding number. We further demonstrate that chiral symmetry breaking in metamaterial crystal chains prevents us from defining a proper topological invariant. We finally numerically confirm, in the microwave domain, the existence of the topological transition in a chiral symmetric metamaterial crystal chain through the study of topological interface modes and their robustness to disorder.
\end{abstract}

DOI: 10.1103/PhysRevB.102.134303

\section{INTRODUCTION}

Currently, one of the most intriguing and exciting concept in condensed matter lies in the recent discovery of topological order [1,2]. Topological insulators [3], especially, have attracted a lot of attention due to their ability to support dissipationless electronic transport on their edges despite the presence of disorder, impurities, and fabrication uncertainties. These developments have lately triggered a search for their classical analogs first in photonics and phononics [4-7], motivated by the exciting possibility to achieve robust backscattering [8] or disorder-immune wave propagation $[9,10]$. Since topology stems from a crystalline-driven effect, previous proposals for classical waves have mainly been based on photonic crystals (PCs) [11] that can be seen as an appropriate platform to mimic topological properties in composite media. PCs indeed reproduce the crystalline nature of materials and exploit a multiple scattering phenomenon on periodic wavelength-scaled structures [12], similar to electron propagation that periodically encounter atoms in crystals [13]. Engineering propagation and topological phase properties in such media hence amounts to carefully designing the microscopic structure of its unit cell to engineer the material spatial dispersion. However, contrarily to atomic lattices, PCs suffer from severe limitations as their wavelength-scale structure makes them inherently diffraction limited and prevent them from being used for low frequency waves.

On the other hand, there is another class of composite media, namely resonant metamaterials (MMs), which are organized at spatial scales much smaller than the wavelength. Their macroscopic propagation properties usually stem from designing the temporal dispersion through modi- fications of the resonant nature of their artificial elementary constituents, the so-called meta-atoms (MAs) [14]. However, the commonly resorted spatially nondispersive (local) homogenization approaches to describe MMs [15] generally prevent us from wholly exploiting the spatial organization of MAs as one potential degree of freedom to tailor MMs properties. They have hence not been fully considered as valuable platforms to study structure-based topology. Yet, the primordial role of the nonlocal response in some sub- $\lambda$ resonant structures was previously underlined, evidencing a strong analogy between the physics of wave propagation in PCs and in most MMs, especially uncovering the importance of multiple scattering at subwavelength scales [16]. Consequently, it was shown that propagation properties of locally resonant metamaterials with polaritonic dispersion relations can be engineered by carefully designing both the unit cell resonant composition and sub- $\lambda$ structure [17]. This work led to defining the novel concept of metamaterial crystals (MMCs): resonant metamaterials with deep sub- $\lambda$ crystalline organization for which both the composition (local or temporal dispersion) and the structure (nonlocal or spatial dispersion) of the unit cell together set the material's macroscopic properties. MMCs then pave the way of transposing and exploiting the tremendous diversity of atomic lattices properties while being the perfectly appropriate platform to bring concepts from solid-state physics to classical waves. This hence opens new perspectives for classical wave propagation control at the deep sub- $\lambda$ scales from both fundamental and applicative points of view, especially regarding topological properties.

Though topology with resonant inclusions was already considered, mainly using tight-binding (TB) lattices of resonators, such studies fail at unleashing the full potential of 
resonant media to uncover topological properties as it only represents a restricted subclass of resonant systems. In the latter indeed, the wave propagation as well as the topological properties are solely based on short-range evanescent couplings between inclusions. It then completely discards the largely underestimated role of the multiple scattering phenomenon as a potential degree of freedom to bring about different topological phases. Consequently, despite dawning studies [18-20], the possibility to achieve topological phases at the sub- $\lambda$ scale by tailoring the metamaterials unit cell structure and the associated new physics have been left widely unexplored. Especially, investigations on topology in MMCs suffer from a severe deficiency of appropriate theoretical tools. Indeed, the minimal definition of topology in resonant media usually relies on a two-band Hermitian tight-binding Hamiltonian, characteristic of evanescent couplings, and that most generally neglects the resonance linewidth of the isolated resonators. Such a model is however obsolete when it comes to topological properties of MMCs, since propagation in the latter is much more complex due to two key features. First, the role of multiple scattering carrying infinite long-range interactions implies no more than two but an infinite number of dispersion bands and second, the locally dispersive response of the MAs cannot be discarded, inducing an intrinsic dispersive response even before considering any periodicity.

In this article we demonstrate that, analogous to evanescently coupled resonator media, the topological properties of one-dimensional MMC chains can be studied by solving a Hermitian eigenvalue problem that appropriately considers the larger complexity of metamaterials presenting both local (resonance) and nonlocal (multiple scattering) dispersion. We further show how this new analytical model can be exploited to study mode symmetry inversions along the bands [20], assess the existence of a topological phase transition, and define a topological invariant, depending on the metamaterial unit cell structural properties. We then evaluate the incidence of chiral symmetry breaking on the different topological phases. Finally, we validate the results evidenced by our model through the numerical study of a topological edge mode at the interface between two MMC chains with different topological nature and explore its robustness to disorder.

\section{DESCRIPTION OF ONE-DIMENSIONAL MMCS FROM A HERMITIAN EIGENVALUE OPERATOR}

Before considering the case of MMCs, let us first briefly remind the current analytical Hamiltonian model that describes topology in resonant lattices with identical neighboring resonators and alternating near-field evanescent couplings, the so-called Su-Shrieffer-Heeger ( $\mathrm{SSH}$ ) chain. It will indeed act as a reference model we will rely on to build an analogous model for MMC chains. For one-dimensional media, the case we focus on in this paper, it consists of solving the following Hermitian eigenvalue problem [Eq. (1)]:

$$
H_{\mathrm{TB}}\left(k_{B}\right)|\Psi\rangle=\omega\left(k_{B}\right)|\Psi\rangle .
$$

Where the tight-binding Hamiltonian $H_{\mathrm{TB}}\left(k_{B}\right)$ [Eq. (2)] is defined for each Bloch wave number $k_{B}$ in the reciprocal space from the resonators intrinsic frequency $\omega_{0}$ and the effective complex coupling strength $K+J e^{j k_{B} A}$ between both inclusions in the unit cell:

$$
H_{\mathrm{TB}}\left(k_{B}\right)=\left[\begin{array}{cc}
\omega_{0} & K+J e^{-j k_{B} A} \\
K+J e^{j k_{B} A} & \omega_{0}
\end{array}\right] .
$$

The Hamiltonian eigenvalues directly set the two bands of the chain dispersion relation $\omega\left(k_{B}\right)$, centered on a limited frequency range around $\omega_{0}$ [Fig. $\left.1(\mathrm{a})\right]$ and the $k_{B}$-dependent associated eigenvectors capture the symmetry of the two corresponding modes. Depending on the relative coupling strength $|K / J|$, the mode symmetry along the band may or may not drastically change, inducing a different topological nature for the chain. This model is extremely convenient and well documented to study topology in many resonant chains and it has been successfully applied to numerous classical systems, in photonics [21], plasmonics [22], polaritonic media [23], or mechanical lattices [24]. Though it only describes a restricted subclass of resonant media, some efforts have been made to extend it to more complex cases. For instance, longerrange interactions have been considered through next nearest neighbor couplings $[25,26]$ or retardation effects, larger scale unit cells have been discussed [27], and local dispersion was introduced through the resonator's polarizability [28] or inducing an additional strong light-matter coupling [29].

Even refined, this model cannot however be applied as it is to MMCs because the physics of wave propagation is inherently different, due to different coupling mechanisms. To underline the fundamental divergences, let us consider the case of a MMC chain that, analogous to the SSH chain, present a dimerized unit cell due to biperiodicity (two different geometrical scales $A$ and $d$ ), bidispersity (two different resonance frequencies $\omega_{1}$ and $\omega_{2}$ ), or both, as schemed in Fig. 1(b). Contrarily to the SSH chain and its two dispersion bands, the dispersion relation of such medium is polaritonic and supports an infinite number of bands [17], whose origin come from two phenomena: first the hybridization between the two resonators in the unit cell and the incoming propagative wave that materialize in the three low frequency bands, and second the infinite long-range multiple scattering interactions of the wave with the periodic structure carried by the propagative field and accounting for the higher Bragg harmonic branches [not represented in Fig. 1(b)]. Hence, instead of dealing with the sole two modes of the SSH chain, we have in MMCs an infinite number of modes for each wave number $k_{B}$. This translates to the fact that each resonator is now not only directly coupled to its two closest neighbors but to every resonator of the chain, through multiple scattering. Moreover, describing this system also requires considering that the problem is highly dispersive, given the frequency dependence of the MAs individual resonant polarizabilities and of the multiple scattering phenomenon (through Green function terms). Both these long-range couplings and local dispersion imply that the propagation in the chain cannot be readily described as a Hermitian eigenvalue problem as in Eq. (1), which is the usual starting point to study the topological properties in standard tight-binding resonators chains. Therefore, it seems crucial to develop an adequate model to understand how the unit cell design can eventually bring about various topological phases in MMCs. 
TIGHT-BINDING NEAREST NEIGHBOR COUPLING

(a)
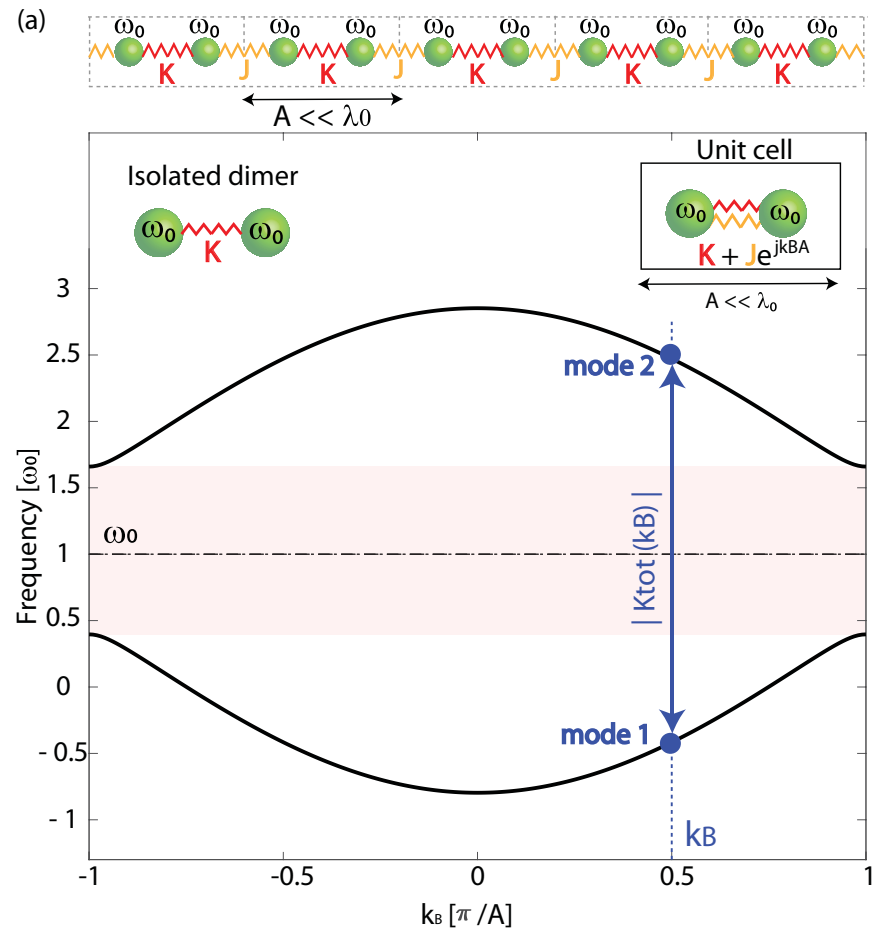

MULTIPLE SCATTERING FAR FIELD COUPLING

(b)
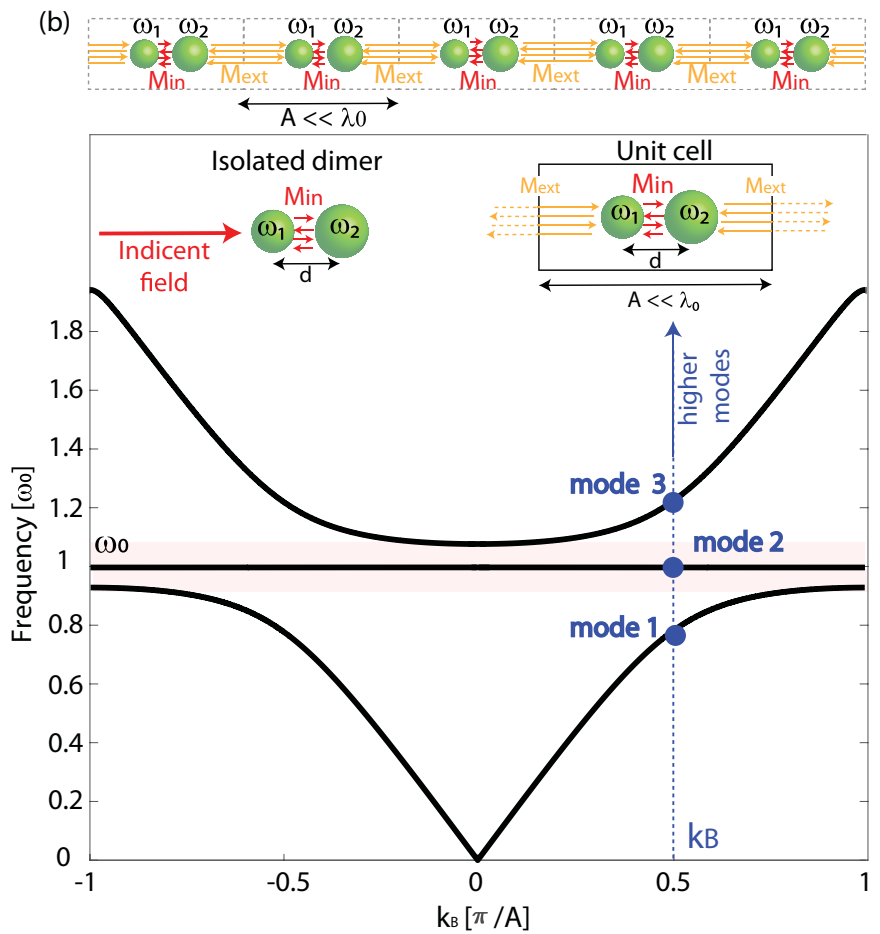

FIG. 1. (a) Scheme of the SSH chain (top) and corresponding dispersion relation (bottom). Isolated dimer of identical resonators coupled through a near field interaction of strength $K$ (inset, left) and coupled resonators dimer in the SSH chain unit cell of subwavelength size $A$ with intra and extra cell couplings respectively $K$ and $J$ (inset, right). (b) Scheme of the MMC chain of asymmetric dimers with intra and extra cell multiple scattering interaction related couplings $M_{\text {in }}$ and $M_{\text {ext }}$ (top) along with the first three bands of the corresponding polaritonic dispersion relation (bottom). Isolated asymmetric dimer of resonators separated by a subwavelength distance $d$, coupled through a propagative incident field conveyed multiple scattering interaction (inset, left) and unit cell of the MMC chain of subwavelength dimension $A$ embedding this dimer (inset, right).

To that aim, we focus on the most general case of dimerized metamaterial crystal chains whose unit cell contains a metamolecule composed of two MAs, with individual resonance frequencies $\omega_{1,2}=\omega_{0} \pm \delta \omega / 2$, which are separated by an arbitrary distance $d<A\left(A \ll \lambda_{0}\right)$ with $\lambda_{0}$ the free space wavelength associated with the resonators mean resonance frequency $\omega_{0}$ and $A$ the chain periodicity [Fig. 1(b)]. We furthermore define the structural dimerization parameter $\delta=2 d / A-1$, which is negative (positive) when the metamolecule dimension $d$ is smaller (larger) than half the unit cell size. We then suggest applying the same strategy as for the tight-binding chain of resonators, which is to start from coupled dipole equations at the scale of the unit cell to derive a matrix formulation that would describe the wave propagation in the chain as an eigenvalue problem. Analog to the SSH model, we consequently start with the isolated unit cell dimer and write the set of two coupled dipole equations for resonators positioned in $x_{1}$ and $x_{2}$ :

$$
\begin{aligned}
& \frac{\omega^{2}}{c^{2}} \alpha_{1}(\omega)^{-1} P_{1}-g(\omega, d) P_{2}=E\left(x_{1}\right), \\
& \frac{\omega^{2}}{c^{2}} \alpha_{2}(\omega)^{-1} P_{2}-g(\omega, d) P_{1}=E\left(x_{2}\right) a,
\end{aligned}
$$

where $\alpha_{1,2}(\omega)=2 c \Gamma /\left(\omega_{1,2}^{2}-\omega^{2}-j \Gamma \omega\right)$ is the lossless polarizability of the each resonator ( $\Gamma$ is the radiation linewidth), $P_{1,2}(\omega)$ its dipolar moment, and $g(\omega, d)=\frac{j c}{2 \omega} e^{j k_{0} d}$ is the
Green function coefficient characterizing the free space lossless propagation between points separated by a distance $d=$ $\left|x_{2}-x_{1}\right|$ with $k_{0}=\omega / c . E\left(x_{1,2}\right)$ is finally the incident field value at the resonators position [Fig. 2(a)]. This leads to the compact form of Eq. (4):

$$
\boldsymbol{H}_{\mathbf{0}}(\omega)\left(\begin{array}{l}
P_{1} \\
P_{2}
\end{array}\right)=\left(\begin{array}{l}
E\left(x_{1}\right) \\
E\left(x_{2}\right)
\end{array}\right)
$$

Resorting to the point scatterer formalism [30], the operator $\boldsymbol{H}_{\mathbf{0}}$ of the isolated dimer is

$$
\boldsymbol{H}_{\mathbf{0}}(\omega)=\frac{j c}{2 \omega}\left[\begin{array}{cc}
1 / t_{1}(\omega) & -e^{-j k_{0} d} \\
-e^{-j k_{0} d} & 1 / t_{2}(\omega)
\end{array}\right] .
$$

Where $t_{1,2}(\omega)=j \omega \alpha_{1,2}(\omega) / 2 c$ is the wave radiated by each resonator after interacting with the incident field. This operator contains the whole physics of the isolated dimer metamolecule, which is the individual resonant responses of both constituting MAs, and their coupling through multiple scattering [Fig. 2(b)]. At this point we can already grasp some fundamental differences with the tight-binding isolated dimer $\left(J=0\right.$ in $\left.H_{\mathrm{TB}}\right)$. First, the far-field coupling that is carried by an incident-propagating wave induces a supplementary term $E(x)$ in Eqs. (3a) and (3b) that prevents us from directly solving the isolated metamolecule as an eigenvalue problem. This expresses the fact that this $2 \times 2$ operator contains information about the interaction between three entities instead of 
(a)

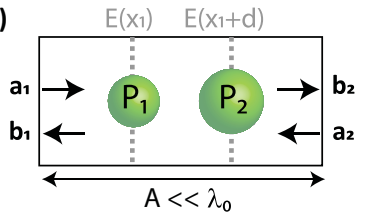

(b)

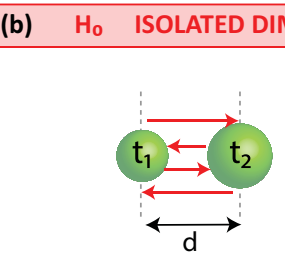

(c) $\mathrm{G}_{\mathrm{A}, \mathrm{d}}$ MULTIPLE SCATTERING
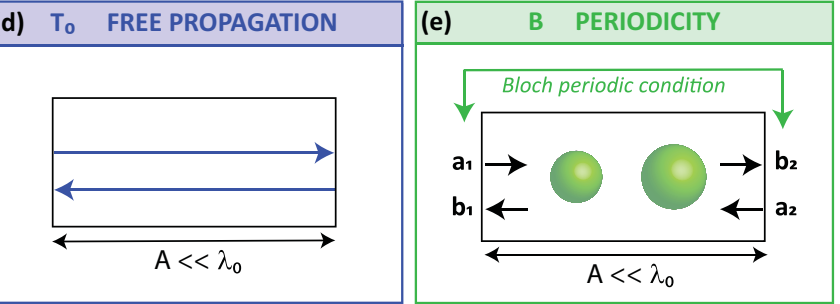

FIG. 2. (a) Unit cell of the studied MMC chain stressing out the resonators dipolar moments $P_{1}$ and $P_{2}$, the incoming and outgoing field amplitude at the unit cell boundaries $\left(a_{1,2}, b_{1,2}\right)$, and the value of the external incident field at the resonators position. Schematic interpretation of the multiple scattering eigenvalue operator $\boldsymbol{H}_{\mathbf{M S}}$ with (b) $\boldsymbol{H}_{\mathbf{0}}$ relating to intradimer multiple scattering (red arrows) and resonators intrinsic polarizabilities included in $t_{1,2}$, (c) $\boldsymbol{G}_{\boldsymbol{A}, \boldsymbol{d}}$ relating the multiple scattering phenomenon between meta-atoms of different unit cells, (d) $\boldsymbol{T}_{\mathbf{0}}$ stemming from a free propagation at the $A$ size unit cell scale, and (e) $\boldsymbol{B}$ accounting for the Bloch periodicity applied to the incoming and outgoing field amplitude at the unit cell boundaries $\left(a_{1,2}, b_{1,2}\right)$. In (c), $g_{ \pm}$stand for the Green function coefficients related to distances $(A \pm d) / 2$.

two in the SSH model, namely the two meta-atoms in the unit cell and an incident plane wave. Second, $\boldsymbol{H}_{\mathbf{0}}$ is not a Hamiltonian since its coefficients are not directly energies. Hence, the antidiagonal Green function term cannot be strictly associated with a coupling between the MAs, as it is the case for the SSH near-field coupling $K$. Finally, we clearly see that each term in $\boldsymbol{H}_{\mathbf{0}}$ is dispersive [the resonant response of individual meta-atoms $t_{1,2}(\omega)$ and the propagation related coefficients], even if there is yet no periodicity, hence no spatial dispersion. This already underlines part of the complexity dealing with MMC chains rather than SSH tight-binding couplings.

Then, from these isolated metamolecule equations, we now want to move on to the periodic infinite chain and see if it could be somehow described as a Hermitian eigenvalue problem. In other words, we want to find out if a Hermitian multiple scattering operator $\boldsymbol{H}_{\mathrm{MS}}\left(\boldsymbol{k}_{\boldsymbol{B}}, \boldsymbol{\omega}\right)$ satisfies

$$
\boldsymbol{H}_{\mathrm{MS}}\left(\begin{array}{l}
P_{1} \\
P_{2}
\end{array}\right)=0 .
$$

It amounts to writing out the equation as a two-body problem while hiding in this new operator the contribution of the incident wave. To do so, we focus on the chain unit cell of size $A$ and call upon the transfer matrix approach, commonly resorted to deal with periodic one-dimensional systems, which links the incoming (outgoing) waves amplitudes $a_{1,2}\left(b_{1,2}\right)$ at the left and right boundaries of the unit cell [Fig. 2(a)]. The incident field at the resonators position in the cell can be extracted from these amplitudes by expressing the Green function matrix $\boldsymbol{G}_{\boldsymbol{A}, \boldsymbol{d}}$ of propagation from the unit cell limits to the resonator's positions $x_{1,2}$ [Fig. 2(c)]:

$$
\left(\begin{array}{l}
E\left(x_{1}\right) \\
E\left(x_{2}\right)
\end{array}\right)=\frac{2 \omega}{j c}\left[\begin{array}{ll}
g\left(\frac{A-d}{2}\right) & g\left(\frac{A+d}{2}\right) \\
g\left(\frac{A+d}{2}\right) & g\left(\frac{A-d}{2}\right)
\end{array}\right]\left(\begin{array}{l}
a_{1} \\
a_{2}
\end{array}\right)=\frac{2 \omega}{j c} \boldsymbol{G}_{\boldsymbol{A}, \boldsymbol{d}}(\omega)\left(\begin{array}{l}
a_{1} \\
a_{2}
\end{array}\right) .
$$

Furthermore, the outgoing field amplitudes $b_{1,2}$ can be decomposed on contributions from the incoming amplitudes $a_{1,2}$ and the waves radiated by each MA that propagate from its position to the cell boundaries:

$$
\left(\begin{array}{l}
b_{1} \\
b_{2}
\end{array}\right)=\boldsymbol{G}_{\boldsymbol{A}, \boldsymbol{d}}(\omega)\left(\begin{array}{l}
P_{1} \\
P_{2}
\end{array}\right)+\boldsymbol{T}_{0}(\omega)\left(\begin{array}{l}
a_{1} \\
a_{2}
\end{array}\right) .
$$

With the free space propagation operator on the unit cell dimension $\boldsymbol{T}_{\mathbf{0}}$ [Fig. 2(d)]:

$$
\boldsymbol{T}_{0}(\omega)=\left[\begin{array}{cc}
0 & e^{j k_{0} A} \\
e^{j k_{0} A} & 0
\end{array}\right] .
$$

In order to uncover an eigenvalue problem [Eq. (6)], we must express the amplitudes $a_{1,2}$ solely from the MA dipolar moments. To do so, we call upon the Bloch condition that accounts for the chain periodicity and implement it to the forward and backward wave amplitudes at the unit cell boundaries [Fig. 2(e)]:

$$
\left(\begin{array}{l}
b_{1} \\
b_{2}
\end{array}\right)=B\left(\begin{array}{l}
a_{1} \\
a_{2}
\end{array}\right) .
$$

With $\boldsymbol{B}$ the Bloch operator and $k_{B}(\omega)$ the MMC chain Bloch wave number

$$
B\left[k_{B}(\omega)\right]=\left[\begin{array}{cc}
0 & e^{-j k_{B}(\omega) A} \\
e^{-j k_{B}(\omega) A} & 0
\end{array}\right] .
$$

Finally, combining Eqs. (4), (7), (8), and (10), we have the following expression independent of the incident propagating wave:

$$
\boldsymbol{H}_{\mathbf{0}}(\omega)\left(\begin{array}{l}
P_{1} \\
P_{2}
\end{array}\right)=\frac{2 \omega}{j c} \boldsymbol{G}_{\boldsymbol{A}, \boldsymbol{d}}(\omega)\left[\boldsymbol{B}\left(k_{B}\right)-\boldsymbol{T}_{\mathbf{0}}(\omega)\right]^{-1} \boldsymbol{G}_{\boldsymbol{A}, \boldsymbol{d}}(\omega)\left(\begin{array}{l}
P_{1} \\
P_{2}
\end{array}\right) .
$$

It leads to the definition of the multiple scattering eigenvalue operator describing the properties of the dimer metamolecule embedded in the infinite MMC periodic chain:

$$
\boldsymbol{H}_{\mathbf{M S}}\left[\omega, k_{B}(\omega)\right]=\boldsymbol{H}_{\mathbf{0}}-\frac{2 \omega}{j c} \boldsymbol{G}_{\boldsymbol{A}, \boldsymbol{d}}\left(\boldsymbol{B}-\boldsymbol{T}_{\mathbf{0}}\right)^{-1} \boldsymbol{G}_{\boldsymbol{A}, \boldsymbol{d}}
$$

The whole physics of the propagation in the MMC chain is included in the compact analytical expression $\boldsymbol{H}_{\mathbf{M S}}$ that expresses how propagation and periodicity act as a perturbation of the isolated metamolecule. We indeed first recognize the isolated dimer operator $\boldsymbol{H}_{0}$ accounting for the resonant response and multiple scattering between both MAs of the unit cell metamolecule [Fig. 2(b)]. The second term comprises the Green function operator $\boldsymbol{G}_{\boldsymbol{A}, \boldsymbol{d}}$ [Fig. 2(c)] relating the multiple scattering phenomenon between MAs from the adjacent unit cells, $\boldsymbol{T}_{\mathbf{0}}$ [Fig. 2(d)] conveys information on the lossless free 
space propagation of the incident wave over the length of the unit cell, and $\boldsymbol{B}$ accounts for the periodicity [Fig. 2(e)].

The multiple scattering operator $\boldsymbol{H}_{\mathbf{M S}}$, despite its relative complex analytical expression, can be numerically estimated knowing the unit cell structure $(A, d)$, MAs resonance parameters $t_{1,2}(\omega)$ (from the point scatterer model [30]), and the infinite periodic chain dispersion relation $k_{B}(\omega)$. The latter [Eq. (14)] can be extracted either by solving $\operatorname{det}\left(\boldsymbol{H}_{\mathbf{M S}}\right)=0$ or more traditionally directly from a transfer matrix approach [31], using the transmission coefficient $T$ of the isolated metamolecule when excited by an incident plane wave [32]:

$$
\cos \left(k_{B} A\right)=\Re\left(\frac{1}{T} e^{-j k_{0}(A-d)}\right) .
$$

Note again that, as for $\boldsymbol{H}_{\mathbf{0}}$ and contrarily to the $\mathrm{SSH}$ model, the multiple scattering eigenvalue operator $\boldsymbol{H}_{\mathbf{M S}}$ is not a Hamiltonian, so that the dispersion relation is not given by the operator eigenvalues. Another fundamental difference with the SSH model lies in the dispersive nature of $\boldsymbol{H}_{\mathbf{M S}}$ that makes it easier to solve the problem for fixed $\omega$ instead of fixing $k_{B}$. Indeed, each frequency is solely associated with two modes $\pm k_{B}(\omega)$, while a given Bloch wave number is linked to an infinity of modes.

We now would like to explore the potential topological behavior of the MMC chains with this dimer metamolecule unit cell. Again, the idea is to refer to the well-documented SSH model and see if some analogy can be derived, despite the physical differences between tight-binding and multiple scattering driven wave propagation, leading to the previously exposed differences in the analytical definition of the eigenvalue problems. Generally, topological properties of a given medium are evaluated from a topological invariant, which is an appropriately defined, potentially symmetry-protected, parameter exhibiting a discontinuous variation at topological phase transitions. In the SSH model, this topological invariant is the bulk winding number $w$. It can be geometrically defined from the vector $\overrightarrow{h_{\mathrm{TB}}}$ stemming from the decomposition of the Hermitian Hamiltonian $\boldsymbol{H}_{\mathbf{T B}}$ on the Pauli matrices and identity matrix [Eq. (15)], which form a base of the real vector space of $2 \times 2$ complex Hermitian matrices:

$$
\boldsymbol{H}_{\mathbf{T B}}\left(k_{B}\right)=h_{0}\left(k_{B}\right) \boldsymbol{I}+h_{x}\left(k_{B}\right) \boldsymbol{\sigma}_{\boldsymbol{x}}+h_{y}\left(k_{B}\right) \boldsymbol{\sigma}_{\boldsymbol{y}}+h_{z}\left(k_{B}\right) \boldsymbol{\sigma}_{z} .
$$

While $h_{0}$ is a simple gauge term and $h_{z}$ is null for chiralsymmetric chains (the most commonly studied topological system, namely the case of identical resonators in the unit cell), the whole information on topology is contained in $\overrightarrow{h_{\mathrm{TB}}\left(k_{B}\right)}=\left[h_{x}\left(k_{B}\right), h_{y}\left(k_{B}\right)\right]$. Due to periodicity, it forms a closed contour as $k_{B}$ spans the reciprocal space, which here simply shapes as a circle. The winding number $w$ is then an integer that geometrically corresponds to the number of times this contour wraps around the origin. In the SSH model, it can take two values ( 0 or 1$)$ depending on the relative coupling strengths $K$ and $J$ and can only change when the band gap closes, for $|K / J|=1$, as any proper topological invariant. When $|K / J|>1(w=0)$, the chain is referred to as topologically trivial while when $|K / J|<1(w=1)$ it is topologically nontrivial [Fig. 4(b)]. The existence of these two different topological phases has a straightforward physical interpretation related to the mode symmetry evolution on the dispersion bands. In chiral-symmetric chains, the latter is solely captured by the phase difference $\phi\left(k_{B}\right)$ between the eigenvectors complex components $u_{1,2}$, related to wave function complex amplitude on both resonators of the unit cell [33]:

$$
\left(\begin{array}{l}
u_{1} \\
u_{2}
\end{array}\right)_{k_{B}}=\left(\begin{array}{c}
e^{j \phi\left(k_{B}\right)} \\
1
\end{array}\right) .
$$

Depending on the topological phase, $\phi\left(k_{B}\right)$ along each of the two dispersion bands can emphasize either a symmetry conservation $(|K / J|>1)$ or a symmetry inversion $(|K / J|<$ 1) while $k_{B}$ varies from 0 to $\pi / A$, as graphically displayed in Fig. 3(a). In topologically trivial chains, each band correspond to a rather monopolar $\left[\phi\left(k_{B}\right)\right.$ close to 0$]$ or rather dipolar $\left[\phi\left(k_{B}\right)\right.$ close to $\left.\pi\right]$ mode. In topologically nontrivial chains, $\phi\left(k_{B}\right)$ varies from 0 to $\pi$ (or inversely) along the two bands. Of course the topological nature of a chain with fixed coupling strengths is no absolute property since it depends on which coupling is considered as intracell or extra cell [Fig. 3(a)], hence strongly pertaining to the elementary unit cell choice.

We now would like to study the case of MMC chains. While the Hermiticity of $\boldsymbol{H}_{\mathrm{MS}}$ is not self-evident, we can however demonstrate that for lossless propagation media and lossless resonators, the operator is Hermitian for $\left(k_{B}, \omega\right)$ pairs corresponding to propagative bands $\left(k_{B} \in \mathbb{R}\right)$ [34]. This property is crucial to explore the potential topological behavior of subwavelength multiple scattering chains of resonators while formally defining a topological invariant, analogous to SSH chains. It alone indeed allows the decomposition of $\boldsymbol{H}_{\mathrm{MS}}$ on the Pauli matrices. We can then define $\overrightarrow{h_{\mathrm{MS}}}\left[\omega, k_{B}(\omega)\right]=$ $\left[h_{0}(\omega), h_{x}(\omega), h_{y}(\omega), h_{z}(\omega)\right]$ the vector resulting from this decomposition that writes as follows, with $k^{ \pm}=k_{0} \pm k_{B}$ :

$$
\begin{aligned}
& h_{0}(\omega)=\frac{j c}{4 \omega}\left\{\frac{1}{t_{1(\omega)}}+\frac{1}{t_{2(\omega)}}-j e^{j k_{0} A}\left[\frac{e^{-j k^{-} A / 2}}{\sin \left(k^{+} A / 2\right)}-\frac{e^{-j k^{+} A / 2}}{\sin \left(k^{-} A / 2\right)}\right]\right\}, \\
& h_{x}(\omega)=\frac{c}{2 \omega}\left\{\sin \left(k_{0} d\right)+\frac{1}{2} \cos \left(k_{0} d\right)\left[\cot \left(\frac{k^{+} A}{2}\right)+\cot \left(\frac{k^{-} A}{2}\right)\right]\right\}, \\
& h_{y}(\omega)=-\frac{c}{4 \omega} \sin \left(k_{0} d\right)\left[\cot \left(\frac{k^{+} A}{2}\right)-\cot \left(\frac{k^{-} A}{2}\right)\right], \\
& h_{z}(\omega)=\frac{j c}{4 \omega}\left[\frac{1}{t_{1(\omega)}}-\frac{1}{t_{2(\omega)}}\right] .
\end{aligned}
$$


(a)
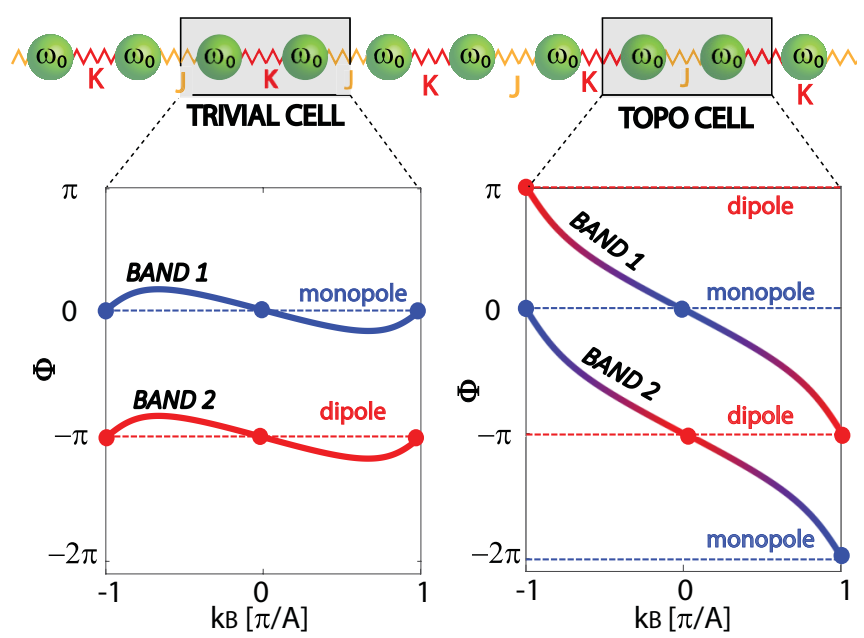

(b)

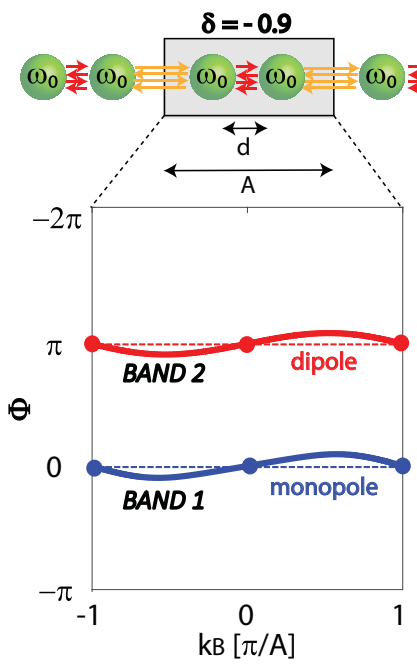

(c)
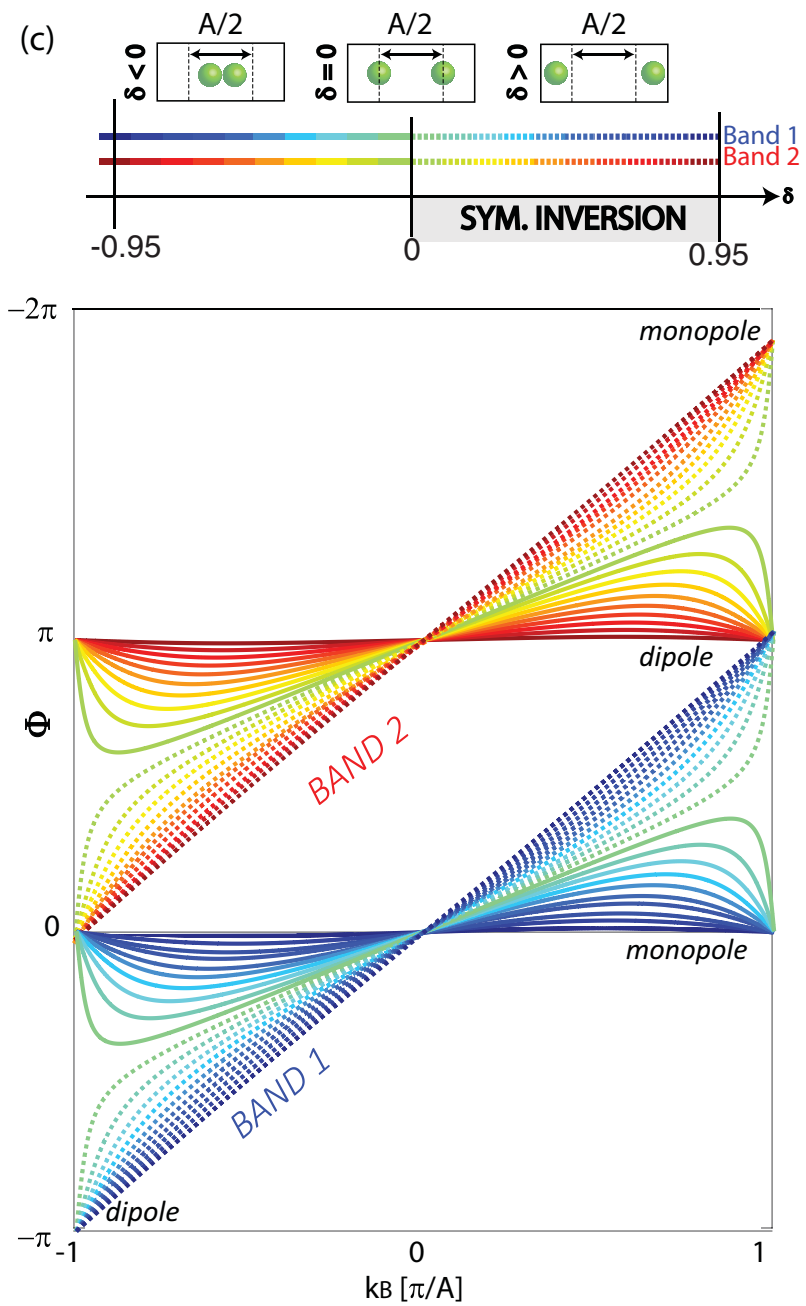

FIG. 3. (a) Phase difference between the dimer resonators of the SSH chain carrying the mode symmetry along the Brillouin zone depending for the trivial (left) and topologically nontrivial (right) unit cells on the two bands of the dispersion relation. Monopolar (dipolar) symmetry is encoded in bluish tones (reddish tones) while pure monopolar (dipolar) symmetry is represented by the blue (red) dots. (b) Same for the chiral MMC chain with a structural dimerization parameter $|\boldsymbol{\delta}|=0.9$ with the phase difference evolution on the first two propagation bands displayed for the $\delta=-0.9$ unit cell (left) and $\delta=0.9$ unit cell (right). (c) Evolution of the mode symmetry along the Brillouin zone for the first (bluish) and second (reddish) band of the MMC dispersion relation when $\delta$ varies from -0.95 to 0.95 . Solid lines related to cases where $\delta<0$ while dashed lines represent cases where $\delta>0$.

Those coefficients have more complex analytical expressions than in the SSH chain. Indeed, we can see that they are highly dispersive, as expected from $\boldsymbol{H}_{\mathbf{M S}}$ dispersive nature. Moreover, contrarily to the SSH chain for which $\overrightarrow{h_{\mathrm{TB}}}$ corresponds to the complex coupling strength between the two resonators of the unit cell, $\overrightarrow{h_{\mathrm{MS}}}$ cannot relate to a simple physical interpretation, though its contour can be as well plotted, as we will see later.

For a more physical insight, the mode symmetry evolution on the dispersion bands, $\left(\begin{array}{c}P_{1}(\omega) \\ P_{2}(\omega)\end{array}\right)$ can as well be extracted from the model, by numerically evaluating the matrix elements $\boldsymbol{H}_{\mathbf{M S}}(1,1)$ and $\boldsymbol{H}_{\mathbf{M S}}(1,2)$ of the multiple scattering eigenvalue operator $\boldsymbol{H}_{\mathrm{MS}}$ :

$$
P_{2}(\omega)=-\frac{\boldsymbol{H}_{\mathbf{M S}}(1,1)\left[\omega, k_{B}(\omega)\right]}{\boldsymbol{H}_{\mathbf{M S}}(1,2)\left[\omega, k_{B}(\omega)\right]} P_{1}(\omega)
$$

Furthermore, we can arbitrarily set $P_{1}\left(\omega, k_{B}\right)=1$, leading to the mode description at each frequency for an arbitrary MMC chain:

$$
\left(\begin{array}{l}
P_{1} \\
P_{2}
\end{array}\right)_{\omega, k_{B}}=\left(\begin{array}{c}
1 \\
\left|P_{2}\left(\omega, k_{B}\right)\right| e^{j \phi\left(\omega, k_{B}\right)}
\end{array}\right) .
$$

In what follows, we use this new analytical model to explore, analog to SSH chains [35], different topological phases that can be achieved in MMC chains. Contrarily to SSH chains however, we do not have access to direct coupling strengths to drive a possible topological transition. We nonetheless know that both structural and resonance properties of the unit cell $\left(\delta, \omega_{1}, \omega_{2}\right)$ shape the wave propagation so that we expect it might as well have an effect on the modes symmetry $\left(\begin{array}{l}P_{1} \\ P_{2}\end{array}\right)$ and on an eventual topological invariant. 


\section{THE MMC BIPERIODIC CHIRAL SYMMETRIC CHAIN}

For the sake of simplicity as well as to relate to the common chiral-symmetric SSH chain, we first focus on chiral-symmetric biperiodic MMC chains $(\delta \neq 0, \delta \omega=0)$ and explore the revealing mode symmetry evolution in the Brillouin zone, depending on the structural dimerization parameter $\delta$. As we know that the topological phase of a given near-field coupled resonators chain strongly depends on the unit cell choice [Fig. 3(a)], we first fix the biperiodic chain structure $|\delta|$ and compare the mode symmetry along the bands as the chain is described from a unit cell containing either the small $(\delta=-|\delta|)$ or large $(\delta=|\delta|)$ dimension metamolecule [Fig. 3(b)]. For the sake of simplicity and since topological properties are mainly defined across a band gap, we here only focus on the first two bands of the dispersion relation. In Fig. 3(b) we display the result for a biperiodic MMC chain with a structural dimerization parameter $|\delta|=0.9$ and MAs resonance set to $\omega_{0}=9.4248108 \mathrm{rad} \mathrm{s}^{-1}\left(\lambda_{0}=2 \mathrm{~m}\right)$. The period is fixed to $A=0.5148 \mathrm{~m}\left(\sim \lambda_{0} / 4\right)$. We can numerically demonstrate that because chirality is preserved, $\left|P_{2}(\omega)\right|=1$ independently of the frequency $\omega$ so that the mode symmetry is only carried in the phase difference $\phi(\omega)$ between the responses of both meta-atoms in the unit cell.

We observe that, analog to the SSH model, the mode symmetry strongly depends on the choice of unit cell. For the $\delta=-0.9$ unit cell indeed, it remains close to a monopole (dipole) as $\phi(\omega)$ sticks close to $0(\pi)$, overall the first (second) band, with pure monopolar (dipolar) symmetry at the Brillouin zone center and edges. This mode symmetry evolution on bands is analog to what is observed in the SSH chain described from the topologically trivial unit cell. On the other hand, describing the chain from the $\delta=0.9$ unit cell emphasizes a symmetry inversion, from monopole to dipole (or inversely) in both first and second dispersion bands as $k_{B}$ spans the Brillouin zone. This behavior seems to relate to the nontrivial topological phase of the SSH chain. Beyond this first observation of different mode symmetry evolution from different chain descriptions, we can further demonstrate that the existence of the symmetry inversion depends on the sign of the dimerization parameter $\delta$. To do so, we observe $\phi$ along the Brillouin zone as the size of the unit cell metamolecule increases, from $\delta=-0.95$ to $\delta=0.95$ [Fig. 3(c)]. The resulting curves are displayed in bluish (reddish) tone for the first (second) band. It shows that as $\delta<0$ (solid lines), the original band symmetry is preserved on each band, while if $\delta>0$ (dotted lines), a symmetry inversion occurs. This behavior, formally evidenced using the chiral-symmetric Hermitian multiple scattering operator, seems to confirm previously numerical and experimental observations $[19,20]$ suggesting that it might be possible, in the MMC chain as in the SSH one, to induce a topological phase transition. We can then presume that the structural dimerization parameter would play a role analog to $|K / J|$ to drive a topological phase transition. However, formally validating this assumption requires two criteria to be met. First, such transition is expected to happen as the band gap between the two studied bands of the dispersion relation closes and second, it must correspond to a discontinuity of a well-defined topological invariant. The first condition is obviously achieved since the symmetry inversion happens as $\delta=0$ which corresponds to the case of a MMC chain with a single meta-atom elementary unit cell of size $A / 2$. Yet describing its dispersion as if it was still biperiodic leads to a folding degeneracy (i.e., band gap closing) at $k_{B}=\pi / A$ [36].

Regarding the topological invariant, we suggest to explore the relevance of the previously defined $\overrightarrow{h_{\mathrm{MS}}}\left[\omega, k_{B}(\omega)\right]$ vector while displaying its contour evolution with $\delta$, analogous to the SSH model. To do so, let us first consider some interesting features that can be stressed from the vector analytical expression in Eqs. (17). First, as in the SSH chain, $h_{0}$ is the decomposition coefficient of $\boldsymbol{H}_{\mathbf{M S}}$ on the identity matrix. It is a simple gauge term that does not influence the chain topological nature. Indeed, while the structural parameter $\delta$ seems to drive the mode symmetry inversion, $h_{0}$ has no direct dependence on $d$ [only an indirect dependence through the dispersion $k_{B}(\omega)$ ]. Moreover, analog to SSH chains, the coefficient $h_{z}$ only depends on the resonant nature of the unit cell meta-atoms and is null when both meta-atoms in the unit cell are identical $\left(\alpha_{1}=\alpha_{2}\right)$, which is equivalent to preserving the chiral symmetry of $\boldsymbol{H}_{\mathbf{M S}}$. The remaining two components $\left[h_{x}(\omega), h_{y}(\omega)\right]$ thus define the topology. As for the SSH model, they lead to a 2D contour, whose dependence on both $d$ and $A$ (hence on $\delta$ ) predicts the key role on defining the MMC chain topological properties. At this point, it is important to stress another substantial difference between $\overrightarrow{h_{\mathrm{TB}}}\left(k_{B}\right)$ and $\overrightarrow{h_{\mathrm{MS}}}\left[\omega, k_{B}(\omega)\right]$. Indeed, the first one is calculated while fixing the wave number $k_{B}$ in the Brillouin zone. Each point of the contour then contains information simultaneously on the two dispersion bands. Hence, there is only one contour to explore the whole properties of the two-band system. In the MMC chain however, the dispersive nature of $\overrightarrow{h_{\mathrm{MS}}}$ makes it easier to observe while fixing $\omega$. A contour is then defined for each dispersion band while spanning its entire frequency range and the whole Brillouin zone is explored by considering the pairs $\left[\omega, \pm k_{B}(\omega)\right]$. Hence, the $\vec{h}$ contour is no longer a global property of the system, but rather a local property of a given band. However, for a given MMC chain described by a given unit cell choice, the information extracted from the contour of different dispersion bands across a band gap is redundant [37] so that we can simply focus on one band.

We choose to plot the contours $\left[h_{x}(\omega), h_{y}(\omega)\right]$ on the first dispersion band as $k_{B}$ spans the reciprocal space for both unit cells $\delta= \pm 0.9$ of the MMC chain of Fig. 3(b) as well as for the presumed transition parameter $\delta=0$ [Fig. 4(a)]. Consistently with Bloch theorem, we observe a closed contour whose shape is however much more complex than the SSH model circles resorting to the corresponding trivial, nontrivial, and transition cases [Fig. 4(b)]. This can be attributed to the multiple scattering operator dispersive nature that makes the contour highly directive, breaking the previous isotropy. It is moreover not straightforward to determine if the contour encloses or not the origin, the geometric condition to define the topological nature of given SSH chains. To overcome this issue, we choose to display the normalized contour $\left(\widetilde{h_{x}}, \widetilde{h_{y}}\right)$ where $\widetilde{h_{x, y}}=h_{x, y} / \sqrt{\left|h_{x}\right|^{2}+\left|h_{y}\right|^{2}}$. As demonstrated for the SSH model [Fig. 4(d)], this amounts to projecting the contour on the unit circle that can be traveled across either totally or partially as the initial contour encloses or not the origin. The 

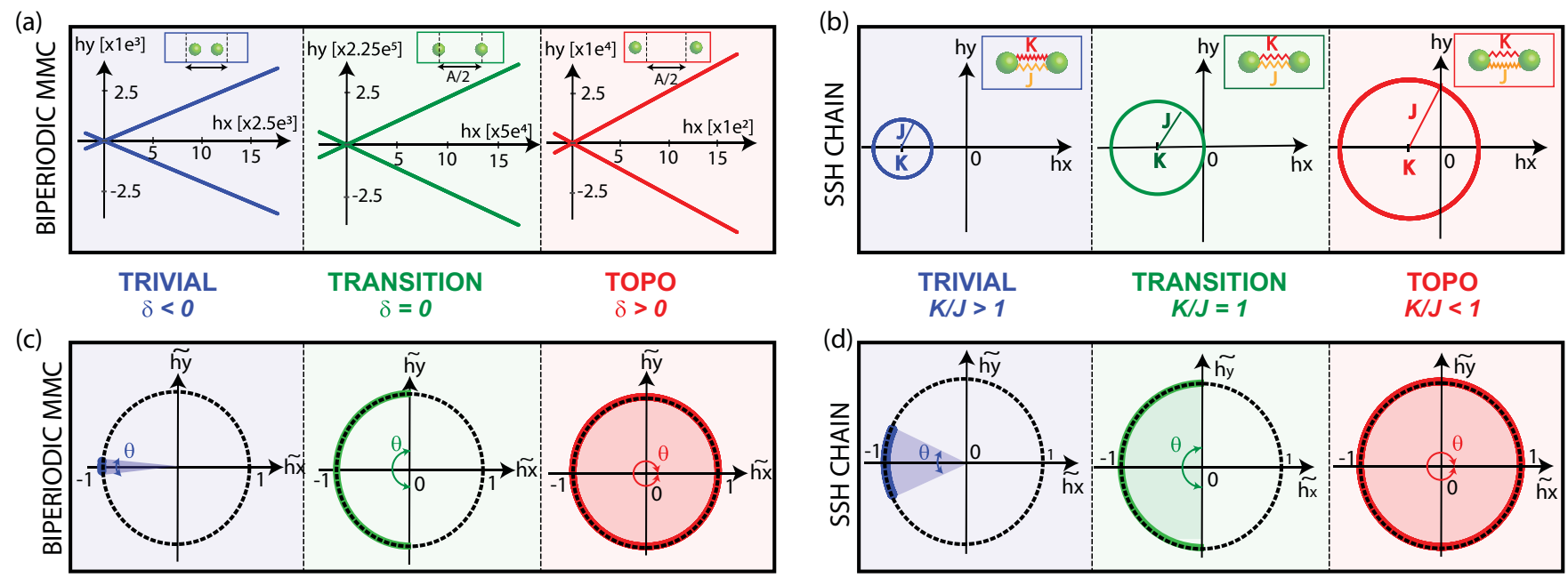

FIG. 4. (a) Vector $\overrightarrow{h_{\mathrm{MS}}}$ plot for the biperiodic chiral MMC chain with structural dimerization parameter $\delta=-0.9$ (blue), $\delta=0$ (green), and $\delta=0.9$ (red). (b) Vector $\overrightarrow{h_{\mathrm{TB}}}$ plot for SSH chains with trivial unit cell $K / J=2$ (blue), topologically nontrivial unit cell $K / J=0.5$ (red) and single resonator unit cell $K / J=1$ (green). (c) Plot of the normalized vector $\overrightarrow{\mathrm{hSS}_{\mathrm{M}}}$ corresponding to a projection of (a) plots on the unit circle (dashed black). (d) Plot of the normalized vector $\overrightarrow{\widetilde{h_{\mathrm{TB}}}}$ corresponding to a projection of (b) plots on the unit circle (dashed black). In (c) and (d), $\boldsymbol{\theta}$ is the angle spanned by the normalized contour in the normalized plane $\left(\widetilde{h_{x}}, \widetilde{h_{y}}\right)$ as the Brillouin zone is explored.

winding number is then null if the spanned angle $\theta \in[0, \pi[$ and 1 if $\theta=2 \pi$. In the case of the biperiodic MMC chain, we observe that, despite the initial contour relative complexity, its normalized counterpart presents the same features as in the SSH chains [Fig. 4(c)]: when $\delta<0, \widetilde{\widetilde{h_{\mathrm{MS}}}}$ maps a portion of the half unit circle so that $\theta<\pi$, leading to a null winding number. Then $\theta$ increases until $\pi$ as $\delta=0$ and finally reaches $2 \pi$ for $\delta>0$, incrementing the winding number to 1 . This winding number, defined analogous to the SSH chain from the decomposition of the multiple scattering operator on the Pauli matrices, analytically confirms the topological phase transition, driven by the structural dimerization parameter $\delta$ in chiral subwavelength MMC biperiodic chains.

\section{THE MMC CHAIN WITH BROKEN CHIRAL SYMMETRY}

Up to now, we only explored the role of the unit cell structure in the topological phase transition, though we know its composition also plays a role in the MMC chain macroscopic propagation properties [17]. We could then legitimately wonder if this additional degree of freedom, namely a finite detuning $\delta \omega$ between the unit cell meta-atoms that amounts to breaking the unit-cell metamolecule chiral symmetry $\left(h_{z} \neq\right.$ $0)$, acts as well on the chain topological nature. Prior to any formal investigation, let us remind the well-documented $\mathrm{SSH}$ case. From this model we know that breaking the mirror symmetry while imposing an in situ potential (i.e., a detuning) prevents us from properly defining the modes symmetry on the monopole/dipole base, making irrelevant the notion of symmetry inversion and a fortiori of topological phases. Hence, modifying the coupling strengths in chiral-symmetry broken SSH chains cannot lead to a topological phase transition. This explains easily resorting to the topological invariant notion because chiral-symmetry breaking imposes a finite nonzero $h_{z}=\omega_{2}-\omega_{1}$, lifting the contour off the $\left(h_{x}, h_{y}\right)$ plane, inhibiting the definition of the winding numbers as a proper topological invariant by prohibiting any band gap closing. The latter indeed requires canceling $\left|\overrightarrow{h_{\mathrm{TB}}}\right|$ (as the contour should cross the origin) for a given $k_{B}$ in the reciprocal space.

Analogous to the $\mathrm{SSH}$ resonators chain, we can intuitively doubt the possibility to induce a topological transition with a nonzero $\delta \omega$ in MMC chains. Indeed, closing the band gap requires canceling $\left|\overrightarrow{h_{\mathrm{MS}}}\right|$, a condition that can only be met, in a given chain, if there is a $\left(\omega, k_{B}\right)$ parameter set for which all components of $\overrightarrow{h_{\mathrm{MS}}}$ are null. Yet from the analytical expression of $\overrightarrow{h_{\mathrm{MS}}}$ [Eqs. (17)], we see that the $h_{z}$ component depends on the unit cell resonators intrinsic complex responses $t_{1}(\omega)$ and $t_{2}(\omega)$. We can show that it never cancels if both resonators have distinct resonance frequencies, whatever the detuning strength, or when it does it does not correspond to a band gap closing [34]. This is physically explained because a necessary condition to close the band gap of a MMC chain described with a two meta-atom unit cell requires that its irreducible unit cell actually contains a single meta-atom, condition that can only happen when $(\delta=0, \delta \omega=0)$, hence totally precluding having two different resonators $(\delta \omega \neq 0)$. This impossibility to drive a topological phase transition in chains with broken chiral symmetry can be illustrated while investigating how the resonator's detuning affects the modes symmetries, which were previously crucial to separate topological phases. Let us for instance consider the previously studied modes of the biperiodic chain $|\delta|=0.9$ whose elementary unit cells $(\delta=$ $\pm 0.9)$ were defined as topologically trivial and nontrivial, and further add a fixed detuning $\delta \omega / \omega_{1}=-0.05$ [Fig. 5(a)]. For each unit cell we monitor the amplitude and phase response $\left[\left|P_{2}\left(\omega, k_{B}\right)\right|, \phi\left(\omega, k_{B}\right)\right]$ of the second meta-atom characterizing the mode symmetry evolution, focusing again on the first band of the dispersion relation [Figs. 5(b) and 5(c)]. We see 

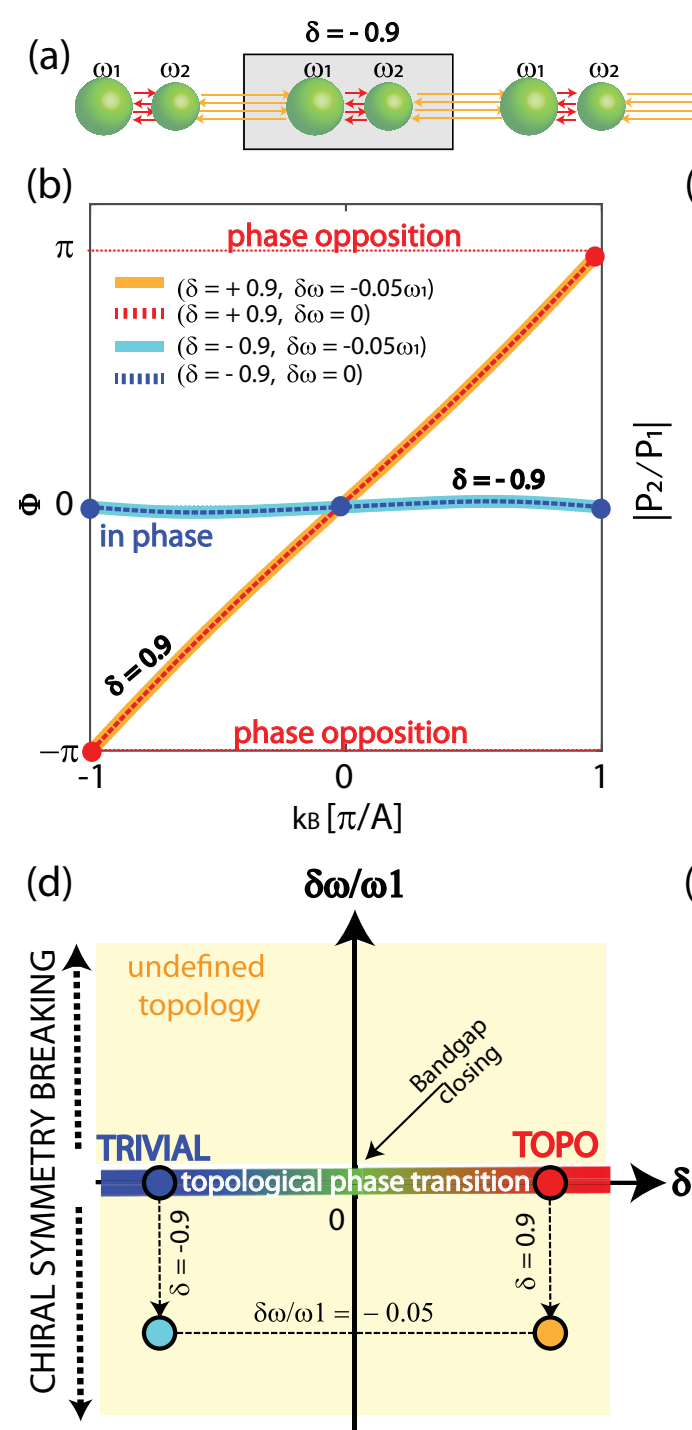

$\delta=0.9$
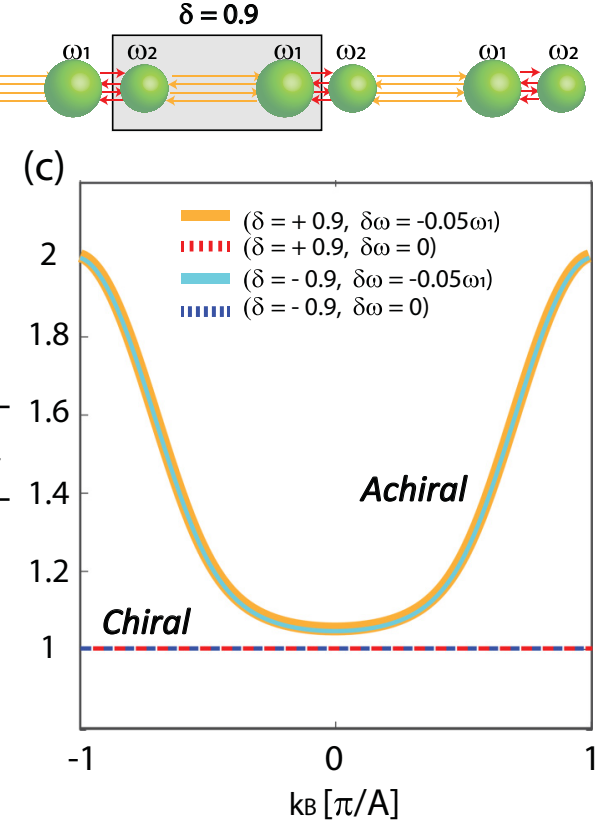

(e)

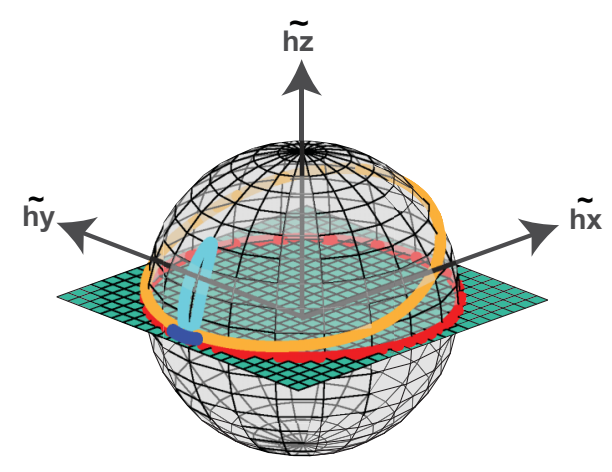

FIG. 5. (a) Scheme of the achiral biperiodic MMC chain with $|\delta| \neq 0$ and $\delta \omega \neq 0$. (b) and (c) Evolution of the mode symmetry on the first dispersion band encoded in the phase difference (b) and amplitude ratio (c) between both resonators of the unit cell for the achiral chain $\delta \omega=-0.05 \omega_{1}$ and $|\delta|=0.9$. The mode symmetry is plotted for both elementary unit cells $\delta=0.9$ (orange) and $\delta=-0.9$ (cyan). As a comparison, the mode symmetry of the chiral chain with equivalent $|\delta|$ is also plotted (dashed blue and red). (d) Scheme of the parameter plane $(\delta, \delta \omega)$ describing all possible two-element unit cell MMC chains. The topological phase transition can occur along the $\delta$ axis where the mode symmetry is properly defined. The colored dots highlight the chains studied in (b) and (c). (e) Normalized $\overrightarrow{\widetilde{h_{\mathrm{MS}}}}$ contour now lying in the $3 \mathrm{D}$ space and projected on the unit sphere for all four studied chains. The chiral chain contours lie as expected in the $h_{z}=0$ plane while breaking the chiral symmetry lifts the contour off the plane.

that while the imposed detuning has no effect on the resonators phase difference [Fig. 5(b)], which depends on the unit cell structural parameter $\delta$ only [38], it however largely impacts the resonators amplitudes, which are no longer equal $\left[\left|P_{2}\left(\omega, k_{B}\right)\right| \neq 1\right.$, Fig. 5(c)]. This prevents the interpretation of the modes as pure monopoles or dipoles, hence making it hard to define a proper symmetry, and a fortiori a symmetry inversion. Hence, breaking the chiral symmetry not only precludes driving a transition because no band gap closing can occur, but also prevents a proper separation of mode symmetries in terms of mode symmetry inversion [Fig. 5(d)]. Another way to emphasize this is to think about the impossibility to geometrically define the topological invariant as a winding number carried by the $\overrightarrow{h_{\mathrm{MS}}}$ contour. As the chiral symmetry is broken and $h_{z}(\omega) \neq 0$, the latter now travels along the three-dimensional $\left[h_{x}\left(k_{B}, \omega\right), h_{y}\left(k_{B}, \omega\right), h_{z}\left(k_{B}, \omega\right)\right]$ parameter space. Though its actual geometric path is not easy to interpret, its normalized counterpart lies at the surface of the unit sphere, as represented in Fig. 5(e) for both unit cells of the $\left(|\delta|=0.9, \delta \omega=-0.05 \omega_{1}\right)$ chain and their chiral counterparts. Here the problem is that, even if we can always mathematically define all sorts of winding numbers (for instance the one around the $\tilde{h}_{z}$ axis), it would not be a proper topological invariant as it would be possible to change its value without closing of the band gap, which can only happen if the contour crosses the point at $\tilde{h}_{x}=\tilde{h}_{y}=\widetilde{h}_{z}=0$, 


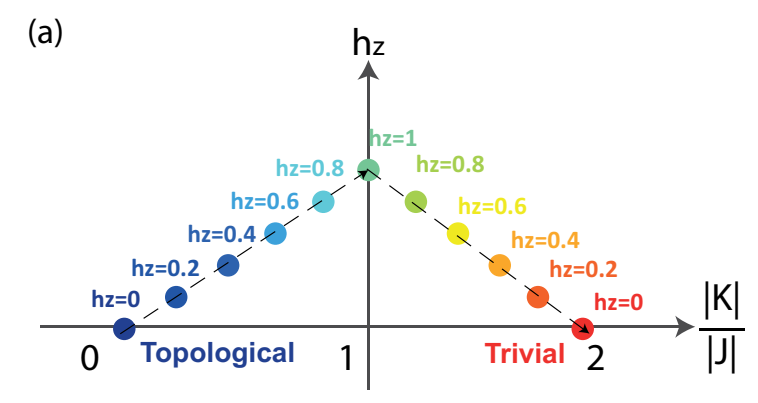

(b)
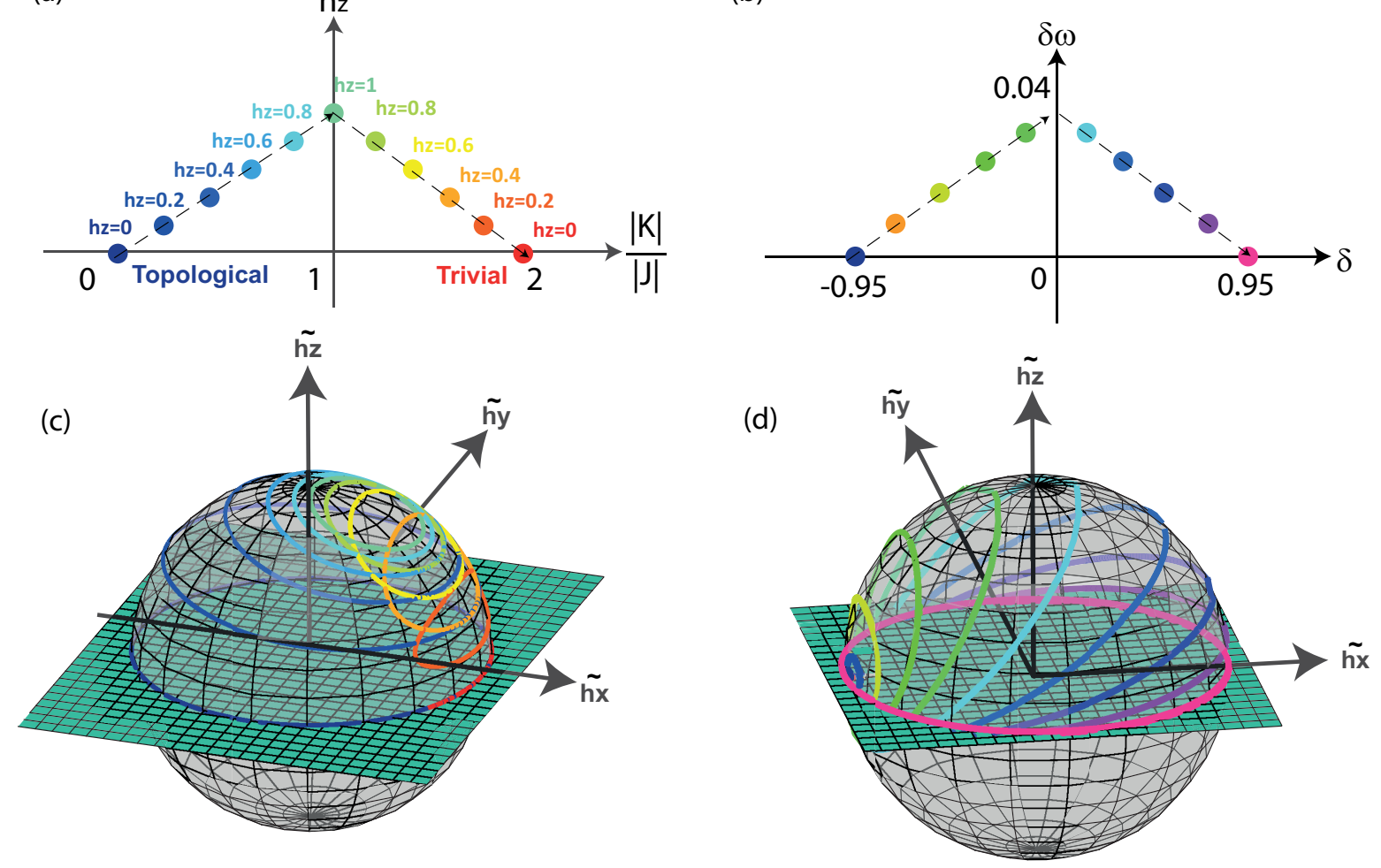

FIG. 6. Example of a topological transition from topologically nontrivial chain (blue) to trivial (red) chain driven without closing the band gap while breaking chirality of the SSH unit cell chain (a) and corresponding plot of the normalized vector $\overrightarrow{\tilde{h}}$ on the unit sphere (b). Analog transition from trivial (red dot) to topologically nontrivial (blue dot) phases induced in a chiral symmetry broken dimerized MMC chain while modifying both the unit cell structure and the dimer detuning (c). (d) Corresponding plot of the normalized vector $\overrightarrow{\widehat{h}_{\mathrm{MS}}}$ on the unit sphere.

an impossible scenario for a closed contour on a sphere. To illustrate this, we provide an example similar to the one known for the tight-binding SSH model, in which a transition from a trivial chiral symmetric chain to a nontrivial one can be driven without closing the band gap by lifting the $\overrightarrow{h_{\mathrm{TB}}\left(k_{B}\right)}$ contour out of its initial $\left[h_{x}\left(k_{B}\right), h_{y}\left(k_{B}\right)\right]$ plane in the $\left[h_{x}\left(k_{B}\right), h_{y}\left(k_{B}\right), h_{z}\left(k_{B}\right)\right]$ parameter space [33]. An example is schemed in Fig. 6(a), where the topological transition is led by varying the coupling ratio $|K / J|$ from 0.1 to 2 while $h_{z}$ simultaneously spans the interval [0:1:0]. As it undergoes the transition from trivial (red) to nontrivial (dark blue), the normalized contour, which now lies at the surface of the unit sphere, never crosses the origin [Fig. 6(c)], avoiding a band gap closing. This chiral symmetry broken transition can as well be implemented in MMC chains while detuning the unit cell resonators meanwhile modifying the unit cell structure, as the example displayed in Figs. 6(b) and 6(d). While $\delta$ shifts from -0.9 to 0.9 , leading the topological transition, $\delta \omega$ slightly lifts the contour off plane transitioning from 0 to 0.04 to 0 .

\section{IMMUNITY OF TOPOLOGICAL EDGE STATES TO DISORDER}

In the previous paragraphs we analytically demonstrated that dimerized MMC chain with preserved chiral symmetry can be attributed a topological nature that can be modified through the unit cell geometrical features. In what follows, we highlight the physical consequences of such topological features in terms of interface mode generation, according to the bulk-edge correspondence $[39,40]$, and observe its robustness against disorder. To do so, we numerically study, using the finite element software COMSOL Multiphysics, a quasi-onedimensional microwave electromagnetic MMC consisting of resonant quarter-wavelength slits made of PEC walls connected to a single mode waveguide. The latter provides the one-dimensional wave propagation that carries the long-range multiple scattering interaction. The slits length and width are, respectively, $h_{0}=3 \mathrm{~cm}$ and $w=1 \mathrm{~mm}\left(w \approx \lambda_{0} / 124\right)$ corresponding to a resonance pulsation $\omega_{0}=15.24 \times 10^{9} \mathrm{~s}^{-1}$. The unit cell of subwavelength size $A=2 \mathrm{~cm}\left(\approx \lambda_{0} / 6\right)$ hosts a metamolecule of size $d$. The studied chain is formed of a topologically trivial MMC subchain composed of $N=41$ cells with structural dimerization parameter $\delta_{1}=-0.2$ followed by an equally long MMC subchain with a structure characterized by $\delta_{2}$ [Fig. 7(a)]. To first evidence the topological transition, we explore the eigenmodes of the finite system around the band gap between the two first bands of the dispersion relation as $\delta_{2}$ varies from -0.9 (trivial MMC) to 0.9 (nontrivial MMC). Indeed, we expect an interface mode to appear as the two subchains exhibit different topological indices. We see in Fig. 7(b) that, while $\delta_{2}<0$, that is when both subchains are topologically trivial, the MMC chain only have eigenfrequencies in the propagative bands. On the other hand, as $\delta_{2}>0$, two new modes appear in the band gap. The first one (red dots) correspond to an interface mode between 

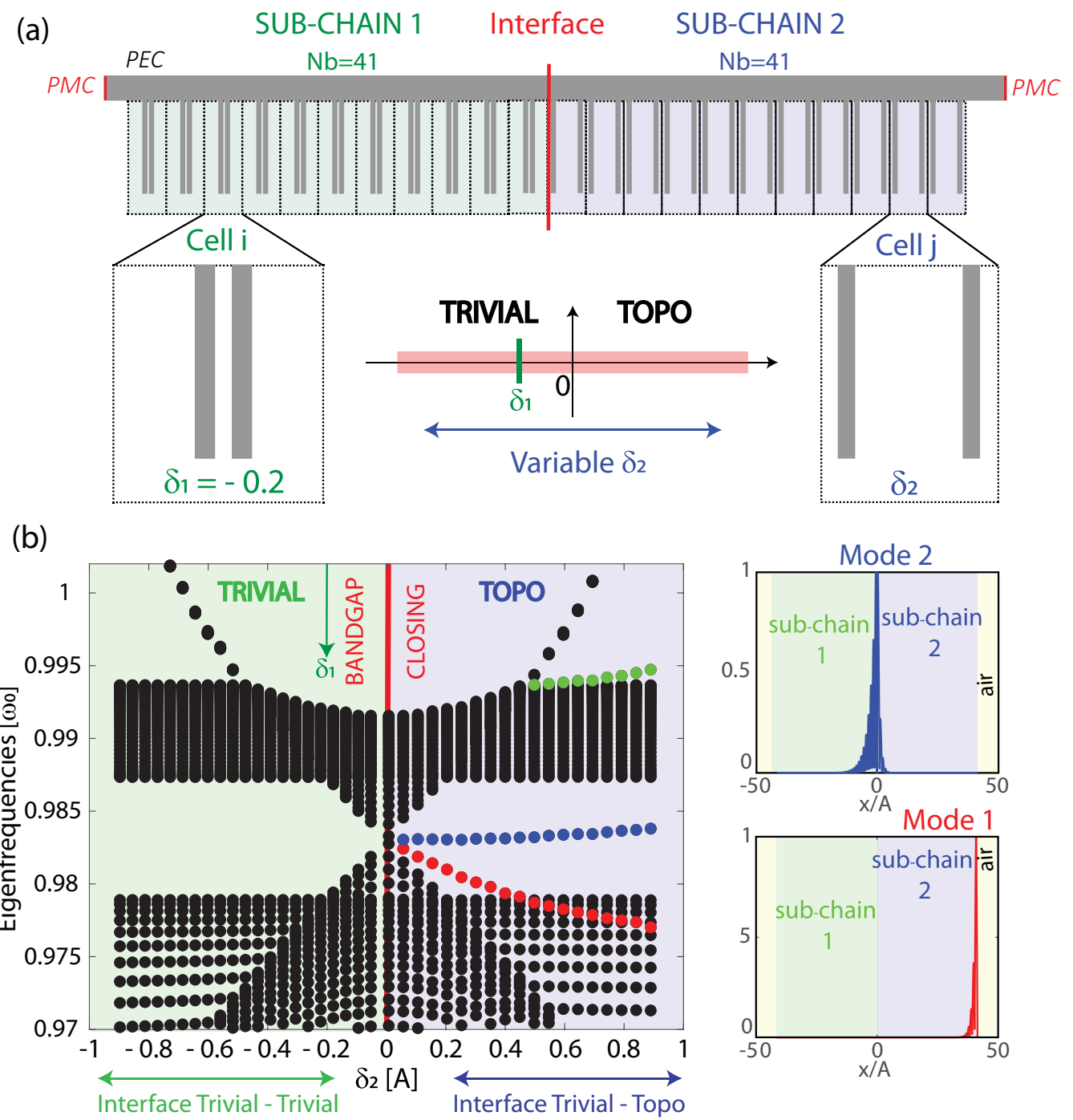

FIG. 7. (a) Scheme of the simulated MMC chain of subwavelength chiral dimeric resonant slits composed of two juxtaposed subchains. Subchain 1 (green area) has in intracell dimer distance $\delta_{1}=-0.2$ corresponding to a topologically trivial phase while subchain 2 (blue area) exhibit a variable distance $\delta_{2}$. The interface between the two subchains is highlighted in red. Boundary conditions at the waveguide sides are perfect magnetic conductor (PMC) and perfect electric conductor (PEC). (b) Eigenfrequencies of the chain in (a) around the first band gap of the polaritonic dispersion relation including the higher modes of the first band and the entire second band. Each column represent a given value of $\delta_{2}$ and green (blue) area stresses the parameter values for which both subchains have the same (different) topological nature. Blue and red dots correspond to modes lying in the band gap whose symmetry is presented in the mode profile insets (right)

the nontrivial subchain and the air-filled waveguide at the right edge of the chain and the second one (blue dots) correspond to a confined interface mode between the two MMC subchains [Fig. 7(b), right]. Both edge mode profiles are represented in insets. It confirms that the second MMC subchain changed its topological nature as the unit cell structural dimerization parameter $\delta$ crosses 0 .

In order to complete the demonstration, we finally verify another classical feature of such interface modes in topological media, namely their ability to be topologically protected and robust to disorder. This property is responsible for the tremendous enthusiasm towards topology, particularly in 2D media to design disorder immune waveguides. In 1D systems, the property could lead to new types of filters with increased robustness to geometrical variations [41]. It is however not valid for every interface mode. For instance, we clearly see in Fig. 7(b) that the mode between the nontrivial subchain and air sinks into the first band of delocalized modes for large enough $\delta_{2}$ values and is therefore a regular (trivial) edge mode with no topological protection. The interface mode between the two subchains with different $\delta$ however always lie in the band gap and we can verify that it remains unaffected while inducing random chirality-preserving disorder levels that do not close the band gap. To do so, we then numerically compute the eigenfrequencies of the MMC chain composed of the topologically trivial subchain $\delta_{1}=-0.2$ juxtaposed to a topologically nontrivial subchain $\delta_{2}=0.2$. Some disorder $\delta d_{i}$ is then added to the intracell metamolecule size $d$, where $\delta d_{i}$ is randomly picked in the interval $[-\sigma ;+\sigma]$ for each unit cell of the MMC chain, $\sigma$ being a fixed disorder strength 
(a)
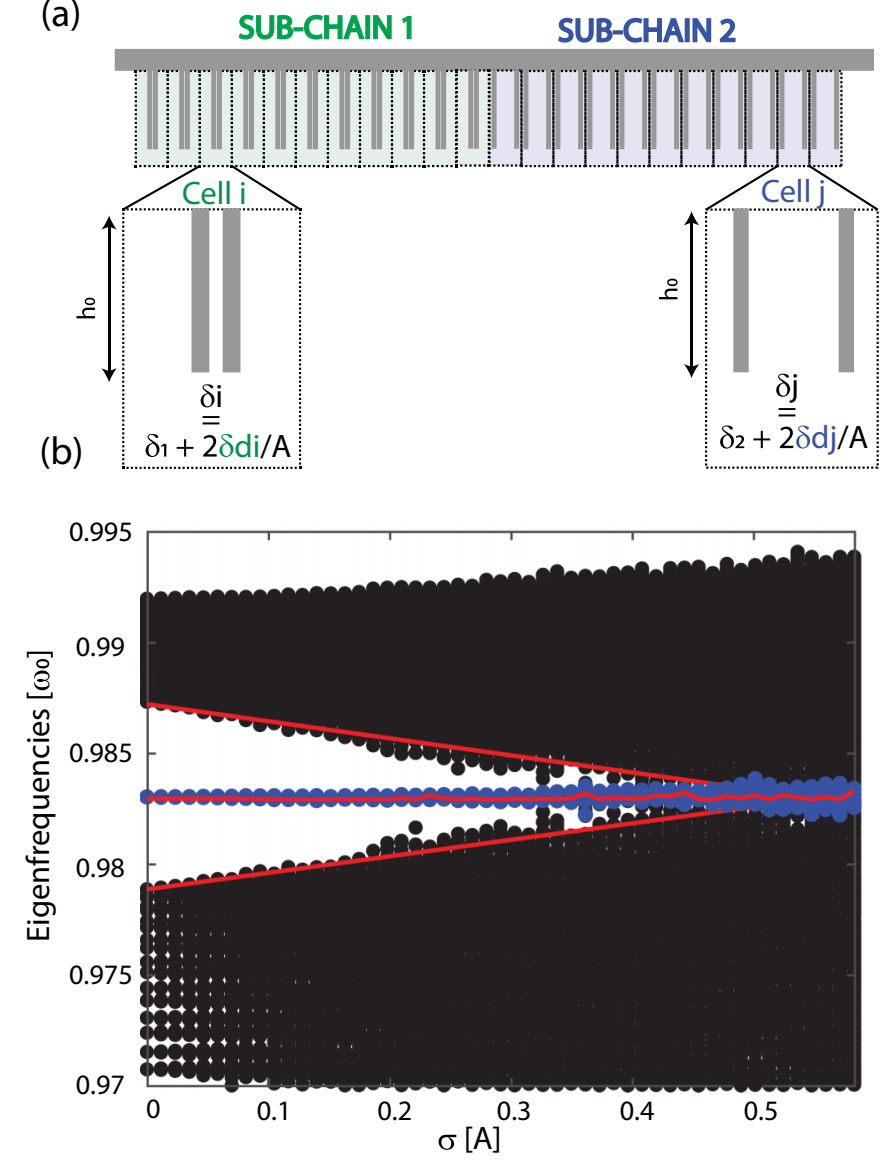

(c)
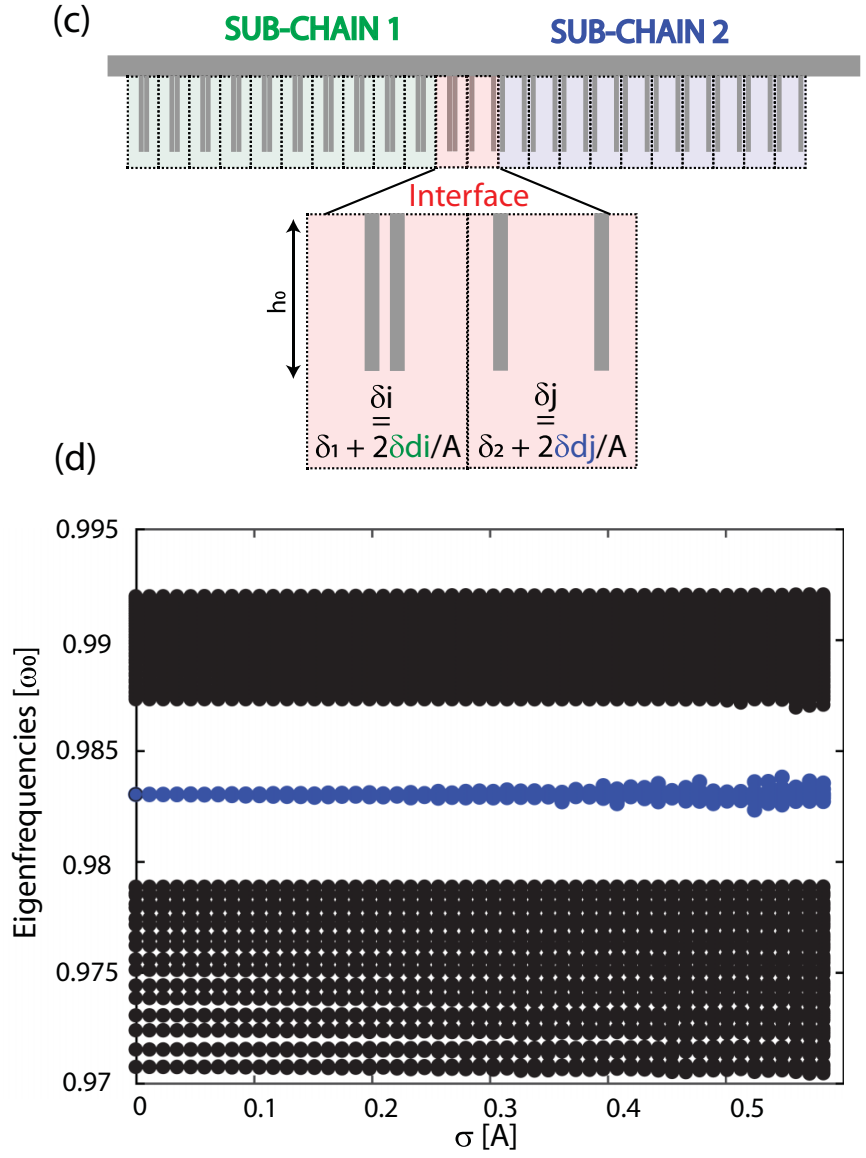

FIG. 8. (a) Scheme of the simulated MMC chain where subchain 1 (2) distance $\delta_{1}\left(\delta_{2}\right)$ is set to $-0.2(0.2)$ and some positional disorder $\delta_{i} \in[-\sigma ;+\sigma]$, with $\sigma$ the disorder strength, is added to each unit cell. (b) Eigenfrequencies of the chain in (a) while varying the disorder strength (averaged over 21 realizations of disorders). Blue dots represent the single interface mode eigenfrequency for each of the 21-disorder realization at constant $\sigma$ (21 dots each $\sigma$ value), which are more or less scattered around the average mode eigenfrequency value (red line). (c) Same as (a) but with positional disorder only added to both interface unit cells (red shaded). (d) Eigenfrequencies of the chain in (c) while varying the disorder strength (averaged over 21 realizations of disorders).

[Fig. 8(a)]. We observe that, when $\sigma=0$ (no disorder), the spectrum shows the two distinct bands of propagative waves and the interface mode stuck in the band gap (blue dot). We then progressively increase the disorder strength, averaging each simulation over 21 realizations of disorder. We see that as the disorder strength increases, propagative bulk modes start filling the band gap, progressively decreasing its width until completely closing it for large enough disorders. In the meantime, the interface mode sticks to the band gap, at a steady frequency despite some slight statistical broadening, if the latter remains opened [Fig. 8(b)]. This is a quite clear signature of the mode topological robustness to disorder, which can be attributed to the different topological nature of the MMC subchains along the interface. Another demonstration of the interface mode robustness consists of applying the positional disorder on the two cells bordering the interface only [Fig. 8(c)]. This amounts to maintaining the MMC subchains properties, hence the band gap opened, but simulating how the interface geometry itself affects the mode eigenfrequency. Figure 8(d) displays the corresponding spectrum as the disorder strength $\sigma$ varies and evidences the robustness of the interface mode eigenfrequency that again sticks to its position within the band gap, confirming its topological protection by the subchains natures.

\section{CONCLUSION}

In this article we investigated the topological properties of one-dimensional metamaterial crystal chains composed of locally resonant scatterers embedded in a propagating medium. In such systems, drastically different from conventional tightbinding scenarios, the propagation depends not only on the meta-atom resonances but also on the unit cell structure, due to the important role of multiple scattering interactions. This makes the analytical study of topological properties in MMC chains much more complex than what has already been studied in resonators chains whose properties are driven by near-field tight-binding interactions, interpreted from the well-known SSH model. Indeed, both the infinite range interactions and the dispersivity of the local resonance and of the multiple scattering phenomenon must be considered. We here however successfully manage to take into account these specificities while deriving a Hermitian eigenvalue problem, analog to the one developed in the well-known SSH model, 
and analytically express a Hermitian multiple scattering operator that contains the whole physics of a MMC chain, including its topological properties. Analog to the winding number, a geometrical topological invariant was emphasized that allowed exploring the structural topological transition in biperiodic chains as well as the impact of chiral-symmetry breaking. Finally, a topological interface mode and its robustness to disorder were numerically studied. We believe that this new model, developed in the simplest case of dimerized one-dimensional chains, helps understanding the role of geometrical and resonance parameters in MMC topological properties. Though it was limited to 1D metamaterials and numerically demonstrated in microwaves, it can be transposed to other frequency ranges or waves as acoustics for instance.
It furthermore paves the way to the study of more complex unit cells in one-dimensional metamaterials that may be investigated using a similar model in order to induce eventual new topological phases and even inspire designs in higher dimension metamaterial.

\section{ACKNOWLEDGMENT}

This project has received funding from the European Union's Horizon 2020 research and innovation programme under the Marie Sklodowska-Curie Grant Agreement No. 798556.
[1] D. J. Thouless, M. Kohmoto, M. P. Nightingale, and M. den Nijs, Quantized Hall Conductance in a Two Dimensional Periodic Potential, Phys. Rev. Lett. 49, 405 (1982).

[2] X.-G. Wen, Topological orders and edge excitations in fractional quantum hall states, Adv. Phys. 44, 405 (1995).

[3] M. Z. Hasan and C. L. Kane, Colloquium: Topological insulators, Rev. Mod. Phys. 82, 3045 (2010).

[4] L. Lu, J. D. Joannopoulos, and M. Soljacic, Topological photonics, Nat. Photonics 8, 821 (2014).

[5] L. Lu, J. D. Joannopoulos, and M. Soljačić, Topological states in photonic systems, Nat. Phys. 12, 626 (2016).

[6] T. Ozawa, H. M. Price, A. Amo, N. Goldman, M. Hafezi, L. Lu, M. C. Rechtsman, D. Schuster, J. Simon, O. Zilberberg, and I. Carusotto, Topological photonics, Rev. Mod. Phys. 91, 015006 (2019).

[7] X. Zhang, M. Xiao, Y. Cheng, M. H. Lu, and J. Christensen, Topological sound, Commun. Phys. 1, 97 (2018).

[8] Z. Wang, Y. Chong, J. D. Joannopoulos, and M. Soljačić, Observation of unidirectional backscattering-immune topological electromagnetic states, Nature (London) 461, 772 (2009).

[9] T. Ma and G. Shvets, Scattering-free edge states between heterogeneous photonic topological insulators, Phys. Rev. B 95, 165102 (2017).

[10] S. Mittal, J. Fan, S. Faez, A. Migdall, J. M. Taylor, and M. Hafezi, Topologically Robust Transport of Photons in a Synthetic Gauge Field, Phys. Rev. Lett. 113, 087403 (2014).

[11] G. Wang, X. Wen, J. Wen, L. Shao, and Y. Liu, Two Dimensional Locally Resonant Phononic Crystals with Binary Structures, Phys. Rev. Lett. 93, 154302 (2004).

[12] J. D. Joannopoulos, S. G. Johnson, J. N. Winn, and R. D. Meade, Photonic Crystals: Molding the Flow of Light (Second Edition) (Princeton University Press, Princeton, NJ, 2008).

[13] C. Kittel, Introduction to Solid State Physics (Wiley, New York, 1996).

[14] N. Engheta and R. W. Ziolkowski (Eds.), Metamaterials: Physics and Engineering Explorations (John Wiley \& Sons/IEEE, New York, 2006).

[15] J. B. Pendry, A. J. Holden, D. J. Robbins, and W. J. Stewart, Magnetism from conductors and enhanced nonlinear phenomena, IEEE T. Microw. Theory 47, 2075 (1999).
[16] F. Lemoult, N. Kaina, M. Fink, and G. Lerosey, Wave propagation control at the deep subwavelength scale in metamaterials, Nat. Phys. 9, 55 (2013).

[17] N. Kaina, F. Lemoult, M. Fink, and G. Lerosey, Negative refractive index and acoustic superlens from multiple scattering in single negative metamaterials, Nature (London) 525, 77 (2015).

[18] S. Yves, R. Fleury, T. Berthelot, M. Fink, F. Lemoult, and G. Lerosey, Crystalline metamaterials for topological properties at subwavelength scales, Nat. Commun. 8, 1 (2017).

[19] Z. Zhang, Y. Cheng, X. Liu, and J. Christensen, Subwavelength multiple topological interface states in one-dimensional labyrinthine acoustic metamaterials, Phys. Rev. B 99, 224104 (2019).

[20] W. Zhu, Y. Q. Ding, J. Ren, Y. Sun, Y. Li, H. Jiang, and H. Chen, Zak phase and band inversion in dimerized one-dimensional locally resonant metamaterials, Phys. Rev. B 97, 195307 (2018).

[21] A. P. Slobozhanyuk, A. N. Poddubny, A. E. Miroshnichenko, P. A. Belov, and Y. S. Kivshar, Subwavelength Topological Edge States in Optically Resonant Dielectric Structures, Phys. Rev. Lett. 114, 123901 (2015).

[22] C. A. Downing and G. Weick, Topological collective plasmons in bipartite chains of metallic nanoparticles, Phys. Rev. B 95, 125426 (2017).

[23] D. D. Solnyshkov, A. V. Nalitov, and G. Malpuech, KibbleZurek Mechanism in Topologically Nontrivial Zigzag Chains of Polariton Micropillars, Phys. Rev. Lett. 116, 046402 (2016).

[24] H. Chen, H. Nassar, and G. L. Huang, A study of topological effects in 1D and 2D mechanical lattices, J. Mech. Phys. Solids 117, 22 (2018).

[25] S. R. Pocock, P. A. Huidobro, and V. Giannini, Bulk-edge correspondence and long-range hopping in the topological plasmonic chain, Nanophotonics 8, 1337 (2019).

[26] L. Li, Z. Xu, and S. Chen, Topological phases of generalized Su-Schrieffer-Heeger models, Phys. Rev. B 89, 085111 (2014).

[27] D. Xie, W. Gou, T. Xiao, B. Gadway, and B. Yan, Topological characterizations of an extended Su-Schrieffer-Heeger model, npj Quantum Inf. 5, 1 (2019). 
[28] C. W. Ling, M. Xiao, C. T. Chan, S. F. Yu, and K. H. Fung, Topological edge plasmon modes between diatomic chains of plasmonic nanoparticles, Opt. Express 23, 2021 (2015).

[29] C. A. Downing, T. J. Sturges, G. Weick, M. Stobińska, and L. M. Moreno, Topological Phases of Polaritons in a Cavity Waveguide, Phys. Rev. Lett. 123, 217401 (2019).

[30] P. De Vries, D. Van Coevorden, and A. Lagendijk, Point scatterers for classical waves, Rev. Mod. Phys. 70, 447 (1998).

[31] P. Markos and C. M. Soukoulis, Wave Propagation: From Electrons to Photonic Crystals and Left-Handed Materials (Princeton University Press, Princeton, NJ, 2008).

[32] See Supplemental Material at http://link.aps.org/supplemental/ 10.1103/PhysRevB.102.134303 for an analytical expression of transmission coefficient (n.d.).

[33] J. K. Asbóth, L. Oroszlány, and A. Pályi, A Short Course on Topological Insulators: Band-Structure Topology and Edge States in One and Two Dimensions (Springer International, New York, 2016).

[34] See Supplemental Material at http://link.aps.org/supplemental/ 10.1103/PhysRevB.102.134303 for the numerical demonstration of $H_{\mathrm{MS}}$ Hermiticity (n.d.).
[35] P. Delplace, D. Ullmo, and G. Montambaux, Zak phase and the existence of edge states in graphene, Phys. Rev. B 84, 195452 (2011).

[36] See Supplemental Material at http://link.aps.org/supplemental/ 10.1103/PhysRevB.102.134303 for dispersion relation plots (n.d.).

[37] See Supplemental Material at http://link.aps.org/supplemental/ 10.1103/PhysRevB.102.134303 for contour plots over the first two dispersion bands of topologically trivial and nontrivial MMC chains (n.d.).

[38] See Supplemental Material at http://link.aps.org/supplemental/ 10.1103/PhysRevB.102.134303 for dependence of mode symmetry on chiral symmetry breaking strength (n.d.).

[39] S. Ryu and Y. Hatsugai, Topological Origin of Zero-Energy Edge States in Particle-Hole Symmetric Systems, Phys. Rev. Lett. 89, 077002 (2002).

[40] R. S. K. Mong and V. Shivamoggi, Edge states and the bulkboundary correspondence in Dirac Hamiltonians, Phys. Rev. B 83, 125109 (2011).

[41] F. Zangeneh-Nejad and R. Fleury, Topological analog signal processing, Nat. Commun. 10, 1 (2019). 